\title{
A Percepção do Enfermeiro na Influência da Hotelaria Hospitalar na Recuperação do Paciente
}

\author{
Maria Lúcia Caires Silval e Júlia Sousa Santos Nunes²
}

\begin{abstract}
Resumo: A hotelaria hospitalar engloba desde como a instituição de saúde desempenha suas funções até como o paciente se sente dentro destes locais e aborda as mudanças da assistência em saúde, pautadas na competitividade, assumindo novos contornos, incluindo no dia a dia os conceitos de gestão profissional. Esta pesquisa foi desenvolvida com o propósito de analisar a percepção do enfermeiro sobre a influência da hotelaria hospitalar na recuperação do paciente em um hospital privado da cidade de Vitória da Conquista na Bahia, cuja característica metodológica foi a de uma pesquisa de campo, de caráter qualitativo e descritiva no ponto de vista dos objetivos da pesquisa. Foi aplicado um questionário para 20 enfermeiros funcionários de um hospital privado onde maioria são do sexo feminino e pós-graduados. O conhecimento científico do profissional facilita na atuação, a preocupação com o cuidado, com a parte física do atendimento ao cliente com o olhar individualizado com pacientes especiais dentre outros. A hotelaria hospitalar também pode trazer benefícios para a qualidade de vida no trabalho da equipe, pois o ambiente torna-se mais alegre, otimizando o processo de trabalho. Os profissionais entrevistados reafirmam também os benefícios da hotelaria hospitalar para os pacientes, onde a humanização se destaca. A hotelaria hospitalar pode trazer benefícios para a qualidade de vida no trabalho da equipe, pois o ambiente torna-se mais alegre, otimizando o processo de trabalho. Os profissionais entrevistados reafirmam também os benefícios da hotelaria hospitalar para os pacientes, onde a humanização se destaca.
\end{abstract}

palavras-chave: Hotelaria hospitalar, humanização, bem estar.

\section{Nurses' Perception about the Influence of Hospital Hospitality Care on Patient Recovery}

\begin{abstract}
The Hospitality Hospitality encompasses how the health institution performs its functions to how the patient feels within these places and addresses the changes in health care, based on competitiveness, taking on new contours, including day-to-day concepts of professional management. This research was developed with the purpose of analyzing the nurses' perception about the influence of hospital hotels in the recovery of the patient in a private hospital in the city of Vitória da Conquista in Bahia, whose methodological characteristic was that of a qualitative field research And descriptive from the point of view of the research objectives. A questionnaire was applied to 20 nurses employed by a private hospital where the majority are female and post-graduate. The scientific knowledge of the professional facilitates in the acting, the concern with the care, with the physical part of the customer service with the individualized look with special patients among others. Hospitality hospitality can also bring benefits to the quality of life in the team work, as the environment becomes more cheerful, optimizing the work process. The professionals interviewed also reaffirm the benefits of hospital hospitality for patients, where humanization stands out. Hospitality hospitality can bring benefits to the quality of life in the team work, as the environment becomes more cheerful, optimizing the work process. The professionals interviewed also reaffirm the benefits of hospital hospitality for patients, where humanization stands out.
\end{abstract}

keywords: Hospitality hospital, humanization, wellness.

\footnotetext{
${ }^{1}$ Graduação em Enfermagem pela Faculdade Independente do Nordeste - FAINOR. Contato: lucaires69@ gmail.com;

;2 Graduação em Enfermagem e Obstétricia pela Universidade Estadual do Sudoeste da Bahia, Pós-graduação em Enfermagem Obstétrica pela Universidade Estadual de Santa Cruz, Pós-graduação em Auditoria de Saúde pela Faculdade Estácio de Sá e Mestrado em Gestão de Saúde pela Universidade Federal da Bahia. Professora da Faculdade Independente do Nordeste - FAINOR.
} 


\section{Introdução}

A hotelaria hospitalar é um tema muito discutido na atualidade, entre gestores hospitalares, uma vez que este conceito engloba como a instituição de saúde desempenha suas funções, destacando também como o paciente se sente dentro destes locais e aborda as mudanças da assistência em saúde, pautadas na competitividade, assumindo novos contornos, incluindo no dia a dia os conceitos de gestão profissional (VENDEMIATTI et al., 2010).

O principal objetivo de um hospital é a prestação de serviços na área da saúde, com qualidade, eficiência e segurança. Mas atualmente por em prática estes três adjetivos vai além dos conceitos tradicionais de cuidado na saúde, pois a sociedade evolui e tais mudanças refletem na administração hospitalar, que desde a década de 1990, passou a sugerir novos conceitos em que tanto o paciente quanto o acompanhante, não se sintam em um hospital, mas como se estivessem hospedados em um hotel. Tal situação é fundamental e, embora pouco discutida em estudos científicos, já demonstram que a qualidade da hotelaria hospitalar influência no processo de cura do paciente (MARQUES; PINHEIRO, 2009).

Assim, é válido destacar que estudos feitos sobre o tema, a exemplo de Passos et al (2013), avultam a contribuição da atividade curativa do paciente, ressaltando que é salutar a implantação de novas abordagens em hospitais, em prol de um atendimento mais humanizado, que supere as expectativas do cliente/paciente dos serviços de saúde e que, além do serviço de saúde, possa fazer o seu papel com cortesia, atenção e presteza, tratando o paciente como cliente e prestando serviços de qualidade como se em um hotel estivessem. Este é um conceito novo, pautado na hospitalidade hoteleira, que busca trazer mais conforto, bem-estar, assistência, segurança e qualidade no atendimento ao paciente.

Sabe-se que a mensuração da qualidade hospitalar envolve um processo complexo, no qual evidenciam-se os interesses dos usuários, dos trabalhadores da saúde, dos acionistas, em se tratando de hospital privado, da rede de fabricantes e distribuidores de insumos, das empresas seguradoras e planos de saúde, dos poderes formalmente constituídos na gerência hospitalar e no governo, e isso demanda contrastes e problemas quando estes não trabalham juntos em prol do melhor resultado final, o que é complicado já que cada um tem interesses diferentes. Neste 
contexto, que envolve a atuação de equipes interdependentes, é necessário destacar que todos devem trabalhar juntos (e não uns contra os outros) para um único objetivo (DIAS, 2005)

A hotelaria hospitalar está diretamente relacionada aos mesmo serviços que são que são oferecidos na hotelaria convencional, mas adaptada ao ambiente hospitalar, com o objetivo de melhorar a condição de estadia do paciente durante a internação (POPP et al., 2007). Segundo Popp et al. (2007), na década de 1980 os planos de saúde começaram a solicitar dos hospitais conveniados que esses tratassem seus usuários com certo diferencial, visando tornar o momento em que o paciente está nas dependências hospitalar mais agradável.

O investimento em alta tecnologia e profissionais cada vez mais qualificados, atendimento de excelência procurando "livrar os hospitais de 'cara de hospital' traz em sua essência uma proposta de adaptação à nova realidade do mercado, modificando e introduzindo novos processos, serviços e condutas"(GONÇALVES; FERREIRA, 2013, p. 161). Os benefícios para quem utiliza o serviço vão desde o social até o físico e psicológico, pois ao estar em um ambiente de segurança e conformo o doente e sua família sentem-se mais acolhidos e o ganho emocional corrobora para o reestabelecimento de sua saúde (GONÇALVES; FERREIRA, 2013).

Almeida (2009) explica que a hotelaria está diretamente relacionada com a empatia que se gera a partir da qualidade oferecida pelos serviços bem como pela forma como os colaboradores da empresa se relacionam com os clientes, no caso do hospital, com os pacientes. Com um mercado cada vez mais competitivo as empresas que lidam com a área da saúde não podem se furtar à mudanças, modernização de equipamentos, colaboradores e gestão. A incorporação desse conceito alavanca o faturamento e traz benefícios aos usurários do sistema. Ainda segundo Almeida (2009, p. 20) "essas mudanças podem ser na arquitetura, decorações, layout, infraestrutura, além de outros aspectos. Implica também em promover tratamentos, capacitação" interna e externa de pessoal.

A mudança no hospital que adere a hotelaria hospitalar deve ser física e nos serviços. Quanto aos aspectos físicos devem ser observados, por exemplo, a acessibilidade, espaços amplos e com iluminação adequada, preferencialmente natural, áreas verdes são sempre muito bem aceitas pelo paciente/cliente e seus familiares. Pelo tempo que normalmente o paciente permanece no ambiente hospitalar sua percepção sensorial torna-se mais sensível, devendo o local estar limpo, bem organizado, colorido e tranquilo (ALMEIDA, 2009). 
O ambiente físico deve proporcionar ao paciente a sensação adequada ao objetivo do hospital que é a promoção da saúde e de uma melhor qualidade de vida, mesmo em um momento tão delicado. $\mathrm{O}$ teto de todo o hospital deve receber atenção especial, pois enquanto aguardam atendimento ou estão hospitalizados os pacientes e seus familiares permanecem muito tempo olhando para cima. Outro aspecto são os ambientes para relaxar e descansar, esses são imprescindíveis a fim de que o acompanhante possa dispor de alguns instantes longe da presença da equipe médica e do familiar hospitalizado (ERHART; BOHRER, 2007).

Erhart e Bohrer (2007) consideram importante observar todos os detalhes da estrutura física do hospital: corredores, recepção, área dos elevadores, escadas, os próprios elevadores, área externa, cores dessas áreas, além dos apartamentos e enfermarias, todos pensados para trazer conforto e segurança aos pacientes e suas famílias, tendo sempre um aspecto de hotel.

Serviços especializados de nutrição e dietética, serviço de apoio, hospitalidade, lavanderia são uma forma de agregar valor à administração hospitalar e contribuem para a atividade curativa do paciente (MARQUES; PINHEIRO, 2009), além disso a hotelaria hospitalar tem como consequência "benefício social, psicológico e emocional para pacientes, familiares e funcionários" (GODOI;2004, p. 40).

\section{Método}

Trata-se de uma pesquisa onde os procedimentos metodológicos se enquadram como pesquisa de campo, sendo por sua natureza qualitativa e descritiva do ponto de vista dos objetivos da pesquisa. Gil (2007) ressalta que a pesquisa descritiva observa, analisa e correlaciona fatos e fenômenos sem manipulá-los. Sendo uma pesquisa de natureza qualitativa, que conforme Minayo (2008), estimula os entrevistados a pensarem livremente sobre um tema, objeto ou conceito e a partir daí mostra aspectos subjetivos e atingem motivações não explícitas, ou mesmo conscientes, de maneira espontânea, sendo utilizada esta pesquisa para obter percepções e entendimento sobre a natureza geral de uma questão, abrindo espaço para a interpretação.

O local escolhido para estudo foi um hospital privado localizado na cidade de Vitória da Conquista-BA. O instrumento utilizado foi uma entrevista por meio de um questionário com 
questões semiestruturadas, constituído de uma parte voltada para caracterização da amostra: idade, tempo de profissão, nível de escolaridade, e outra parte relacionada aos objetivos da pesquisa, tais como: conhecimento acerca da hotelaria hospitalar e opiniões a respeito do tema relacionado também a esta e sua influência na recuperação do paciente, entre outras.

A coleta de dados foi realizada entre os meses de abril e maio de 2017. Cada participante foi identificado pela abreviação do nome enfermeiro e por número, sendo que o primeiro profissional entrevistado foi identificado como Enf. 01 até o último profissional entrevistado identificado como Enf. 20.

Os participantes da pesquisa tiveram garantia de sigilo, podendo desistir a qualquer momento e em qualquer fase da pesquisa. Em respeito à resolução 466/12 do Conselho Nacional de Saúde utilizou-se o Termo de Consentimento Livre Esclarecido (TCLE), onde destacará o objetivo e consequência da pesquisa. As informações colhidas foram lidas e examinada de forma detalhada com análise descritiva, identificando as respostas que trouxeram informações para gerar informação. O projeto foi aprovado pelo Comitê de Ética e Pesquisa da Faculdade independente do Nordeste.

\section{Resultados e Discussão}

A tabela a seguir, elenca características utilizadas para traçar o perfil sociodemográfico dos profissionais entrevistados onde teve um destaque quando $90 \%$ destes são do sexo feminino e apenas $10 \%$ são do sexo masculino.

Tabela 1 - Dados sociodemográficos de usuários de um Hospital privado de Vitória da Conquista - BA.

Dados Sociodemográficos dos Participantes

\section{Totais Parciais}

$\%$

\section{Gênero}

Masculino

02

10

Feminino

18

90 
Id on Line Revista Multidisciplinar e de Psicoloqia

Id on Line Multidisciplinary and Psycology Journal

\section{Idade}

18 a 28 anos

29 a 38 anos

39 a 48 anos

49 a 59 anos

\section{Escolaridade}

Superior Completo

Pós Graduado

Mestrado

Doutorado

\section{Tempo de}

\section{Profissão}

Menos de 3 anos

Entre 3 a 9 anos

Entre 9 a 15 anos

Mais de 15 anos

\footnotetext{
Fonte: as autoras
}

Desde o início a arte de cuidar era vista como ato feminizado, e foi criando uma certa resistência para o público masculino em se especializarem na área de enfermagem. No Brasil, os primeiros homens enfermeiros surgiram depois da implantação de hospitais psiquiátricos, onde era necessário ter maior força física e com isso os homens passaram a assumir cargos na área, porém a participação masculina ainda é considerada pouca na enfermagem, que ainda ser vista como uma profissão reconhecida como do sexo feminino ainda causam uma insensibilidade para os homens quanto na escolha da profissão (SOUSA,214).

Quanto à idade dos profissionais, a pesquisa mostrou uma maioria de faixa etária entre 18 a 28 anos, com $55 \%$ no total.

Em relação ao nível de escolaridade 55\% deles são pós graduados, $40 \%$ só tem o nível superior, 5\% tem um mestrado e nenhum dos pesquisados tem o nível de doutorado. 
A pesquisa realizada possibilitou a obtenção da percepção dos enfermeiros sobre a hotelaria hospitalar. Quando indagadas sobre o que é hotelaria hospitalar, pode-se observar que a maioria dos enfermeiros não conheciam essa modalidade de atendimento, embora salientaram ser um serviço inovador e de grande benefício para os usuários. Por ser um tipo de atendimento criado recentemente é aceitável o desconhecimento desses profissionais, mas sem esquecer a necessidade constante de atualização e capacitação.

Podemos observar na fala da Enf. 07, onde a mesma afirma que a hotelaria hospitalar "é um setor responsável pelos leitos, organização e gerenciamento de rotatividade de um hospital". A profissional relaciona a hotelaria hospitalar com a organização dos leitos em uma enfermaria, por exemplo, que não é o objetivo da hotelaria hospitalar.

Observou-se que uma pequena parcela dos entrevistados já haviam se atualizado sobre o tema onde esclareceram que a hotelaria hospitalar, tem como objetivo abranger "vários outros serviços que possam oferecer conforto e qualidade, incluindo lavanderia, portaria, nutrição" Enf. 01, Enf. 03, Enf. 11 e Enf.18.

Foram abordados os componentes da hotelaria hospitalar. Como abordado acima, vários serviços podem ser adaptados em um hospital. Dentre eles, a recepção, alimentos e bebidas, serviços de quarto. No gráfico 01 , podemos observar quais dos componentes foram destacados pelos entrevistados.

Gráfico 01 - Componentes que fazem parte da hotelaria hospitalar

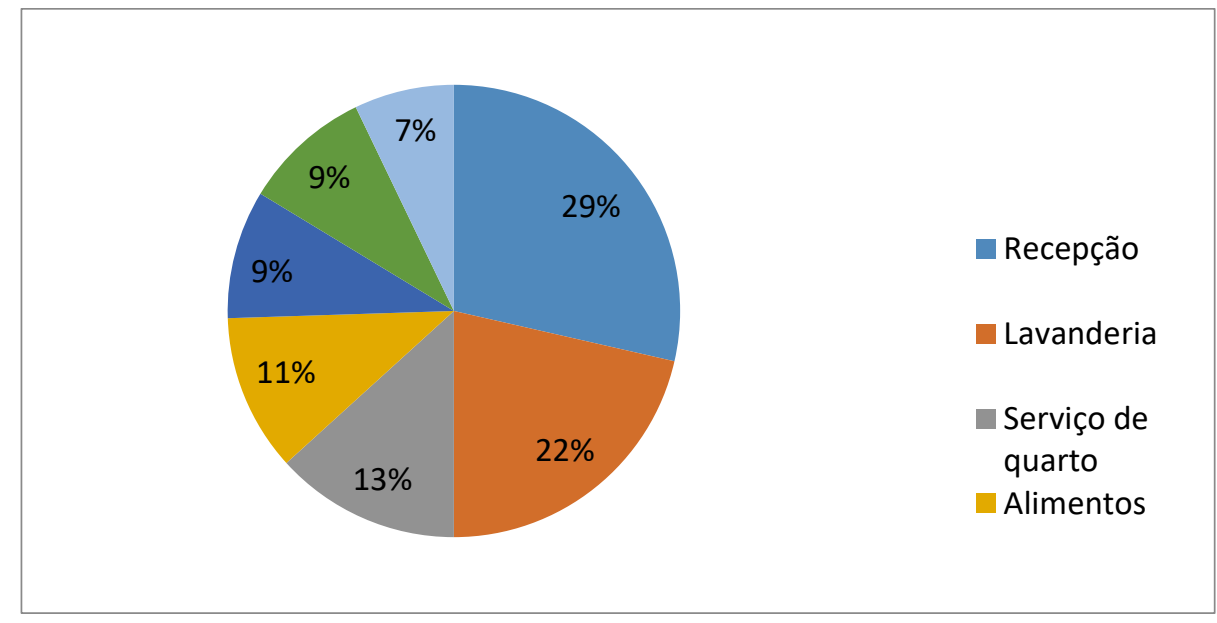

Fonte: as autoras 
Quando indagados sobre os componentes que fazem parte da hotelaria hospitalar, ao participantes da pesquisa, todos responderam ao menos um dos componentes.

Segundo Popp et al. (2007) todos os colaboradores do hospital, e não apenas equipe médica, devem estar envolvidos no modelo de hotelaria, os métodos e modo de lidar com o cliente/paciente deve seguir o modus operandi de um hotel, devendo a equipe gestora estar ciente de todos as particularidades do serviço, como os citados a seguir: Serviços da hotelaria adaptáveis à atividade hospitalar, Recepção, Concièrge (balcão de informações), Alimentos e bebidas, Lavanderia, Reservas, Mensageiros e capitão-porteiro, Governança (governanta e camareira), Serviço de quarto, Restaurantes, Departamento de eventos (seminários, congressos, cursos e reuniões), Salas de lazer (salas de leitura, brinquedotecas, jogos, música e bibliotecas).

A mudança no hospital que adere a hotelaria hospitalar deve ser física e nos serviços. Quanto aos aspectos físicos devem ser observados, por exemplo, a acessibilidade, espaços amplos e com iluminação adequada, preferencialmente natural, áreas verdes são sempre muito bem aceitas pelo paciente/cliente e seus familiares. Pelo tempo que normalmente o paciente permanece no ambiente hospitalar sua percepção sensorial torna-se mais sensível, devendo o local estar limpo, bem organizado, colorido e tranquilo (ALMEIDA, 2009).

O ambiente físico deve proporcionar ao paciente a sensação adequada ao objetivo do hospital é a promoção da saúde e de uma melhor qualidade de vida, mesmo em um momento tão delicado. O teto de todo o hospital deve receber atenção especial, pois enquanto aguardam atendimento ou estão hospitalizados os pacientes e seus familiares permanecem muito tempo olhando para cima. Outro aspecto são os ambientes para relaxar e descansar, esses são imprescindíveis a fim de que o acompanhante possa dispor de alguns instantes longe da presença da equipe médica e do familiar hospitalizado (ERHART; BOHRER, 2007).

Erhart e Bohrer (2007) consideram importante observar todos os detalhes da estrutura física do hospital: corredores, recepção, área dos elevadores, escadas, os próprios elevadores, área externa, cores dessas áreas, além dos apartamentos e enfermarias, todos pensados para trazer conforto e segurança aos pacientes e suas famílias, tendo sempre um aspecto de hotel. Serviços especializados de nutrição e dietética, serviço de apoio, hospitalidade, lavanderia são uma forma de agregar valor à administração hospitalar e contribuem para a atividade curativa do paciente (MARQUES; PINHEIRO, 2009), além disso a hotelaria hospitalar tem como 
consequência "benefício social, psicológico e emocional para pacientes, familiares e funcionários" (GODOI;2004, p. 40).

Gráfico 2 - Sobre a influência da hotelaria hospitalar na recuperação do paciente, os profissionais entrevistados destacaram os benefícios do ambiente hoteleiro no hospital, como vemos abaixo:

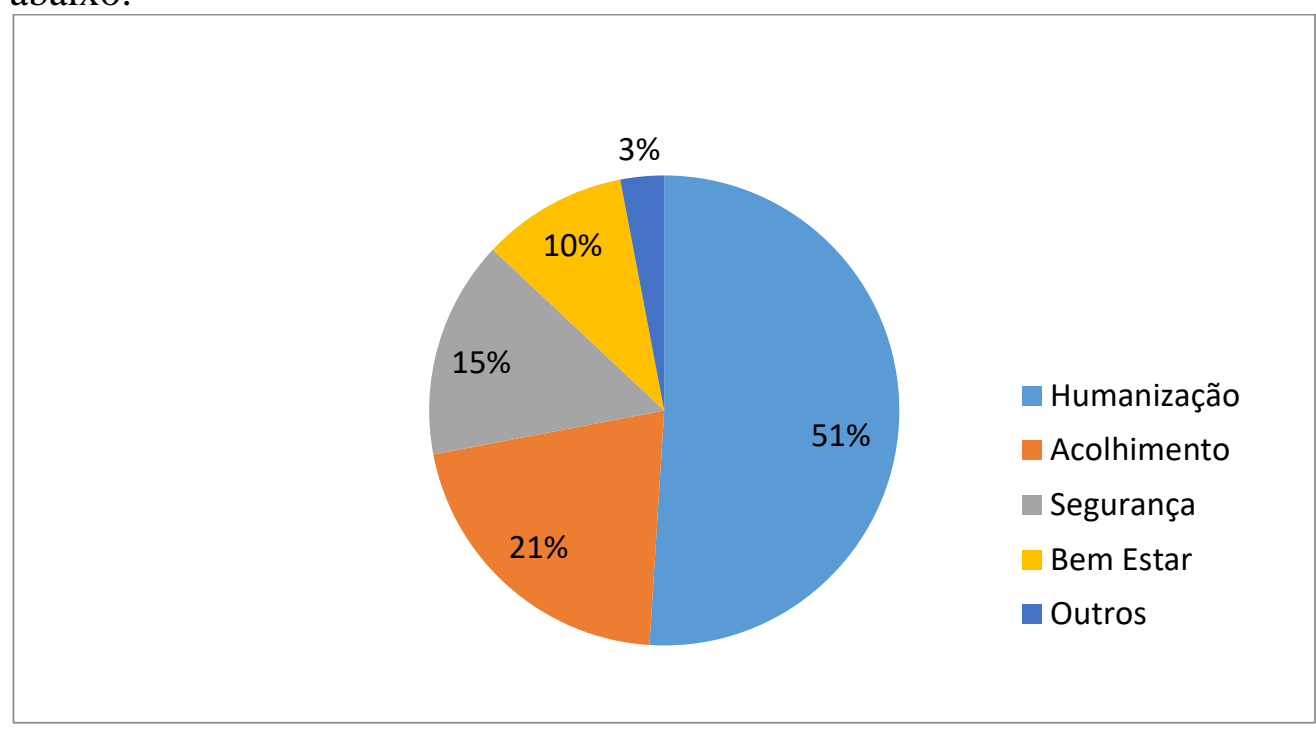

Fonte: as autoras

A hotelaria hospitalar também pode trazer benefícios para a qualidade de vida no trabalho da equipe, pois o ambiente torna-se mais alegre, otimizando o processo de trabalho. Os profissionais entrevistados reafirmam também os benefícios da hotelaria hospitalar para os pacientes, onde a humanização se destaca, como no relato da Enf. 4, que destaca a "qualidade do serviço, retorno, satisfação do cliente, melhoria contínua".

A Enf. 15 afirma "atenção especial [...] o paciente acomoda-se bem tornando o ambiente mais acolhedor, mais próximo do retrato da sua residência”.

A estratégia de hotelaria tem também por objetivo propiciar uma imagem renovada ao hospital que antes era visto como um ambiente frio e pouco preocupado com o bem-estar do paciente. O conforto e a segurança passam a ser as metas principais dos hospitais que aderiram a hotelaria em seu serviço. Vale salientar que hotelaria hospitalar foi aderida apenas por parte dos hospitais particulares brasileiros, visto que a rede pública tem gestão e investimento deficitário (GONÇALVES; FERREIRA, 2013). 


\section{Considerações Finais}

Dos enfermeiros entrevistados grande maioria são do sexo feminino e pós graduados. Observou-se na pesquisa que grande parte dos enfermeiros não tem um conhecimento completo sobre a hotelaria hospitalar. O conhecimento científico do profissional facilita na sua atuação, a preocupação com o cuidado, com a parte física do atendimento ao cliente, o olhar individualizado com pacientes especiais dentre outros, é de fundamental importância.

A hotelaria hospitalar pode trazer benefícios para a qualidade de vida no trabalho da equipe, pois o ambiente torna-se mais alegre, otimizando o processo de trabalho. Os profissionais entrevistados reafirmam também os benefícios da hotelaria hospitalar para os pacientes, onde a humanização se destaca. Destaca-se ainda a necessidade de maior esclarecimento sobre a hotelaria hospitalar para que os funcionários passem a conhecer mais sobre o atual tema.

\section{Referências}

ALMEIDA, Maria Nathalia de Brito Coelho. Hotelaria hospitalar: Cultura e hospitalidade no atendimento a idosos. 2009. Monografia (Especialista em Hotelaria Hospitalar). Universidade de Brasília, Brasília, 2009.

DIAS, MMA. Enfermagem e hotelaria hospitalar na promoção da hospitalidade. [Dissertação]. São Paulo: Universidade Anhembi Morumbi; 2005. Disponível em: http://www.livrosgratis.com.br/download_livro_29313/enfermagem_e_hotelaria_hospitalar_n a_promocao_da_hospitalidade.

ERHART, A. C.; BOHRER, J. O. Serviços de Hotelaria Hospitalar: uma abordagem prática para implantação. Trabalho de Conclusão de Curso. Universidade do Sul de Santa Catarina. Florianópolis, 2007.

GODOI, A. F. Hotelaria Hospitalar e Humanização no Atendimento em Hospitais: pensando e fazendo. 1. ed. São Paulo: Ícone, 2004.

GONÇALVES, Islaine Cristiane Oliveira; FERREIRA, Lissa Valeria Fernandes. Gestão de hotelaria hospitalar: percepções efundamentos. Turismo: Estudos \& Práticas (RTEP/UERN), Mossoró/RN, vol. 2, n. 2, jul./dez. 2013. 
MARQUES, Melissa; PINHEIRO, Mirian Teresinha. A influência da qualidade da hotelaria hospitalar na contribuição daatividade curativa do paciente. Revista Anagrama - Revista Interdisciplinar da Graduação. Ano 2 - Edição 3 - Março-Maio de 2009.

PASSOS, Xisto Sena; RIBEIRO, Ana Caroline Silva; IGNÁCIO, Ana Cristina; LEMOS, Isabela Castro. Hotelaria hospitalar: buscando inovação no padrão de atendimento ao cliente através da humanização. Artigo apresentado ao Curso de MBA - Administração Hospitalar e Marketing na Saúde do Centro de Estudos de Enfermagem e Nutrição/Pontifícia Universidade Católica de Goiás, 2013.

POPP, Elisabeth Victória et al. Hotelaria e hospitalidade. Ed. Ver. E ampl. São Paulo> IPSIS, 2007.

SOUZA, Airle Miranda de; MOURA, Danielle do Socorro Castro; CORRÊA, Victor Augusto Cavaleiro. Implicações do pronto-atendimento psicológico de emergência aos que vivenciam perdas significativas. Psicologia: ciência e profissão, v. 29, n. 3, p. 534-545, 2009.

SOUZA, Leonardo Lemos de et al. Representações de gênero na prática de enfermagem na perspectiva de estudantes. Ciências \& Cognição, p. 218-232, 2014. Disponível em: < file:///C:/Users/Gil/Downloads/908-6898-1-PB.pdf>.

VENDEMIATTI, Mariana et al. Conflito na gestão hospitalar: o papel da liderança. Ciênc. saúde coletiva, Rio de Janeiro, v. 15, supl. 1, p. 1301-1314, 2010.

\section{Como citar este artigo (Formato ABNT):}

SILVA, Maria Lúcia C.; NUNES, Júlia S.S. A Percepção do Enfermeiro na Influência da Hotelaria Hospitalar na Recuperação do Paciente. Id on Line Revista Multidisciplinar e de Psicologia, Abril de 2017, vol.11, n.35, p.168-179. ISSN: 1981-1179.

Recebido: 17.05.2017

Aceito: 22.05 .2017

Anexo 


\section{QUESTIONÁRIO}

Profissional: Idade:

Tempo de profissão: Nível de escolaridade:

Dados pessoais:

1. Gênero:

( ) Masculino

( ) Feminino

2. Idade:

( ) 18 a 28 anos

( ) 29 a 38 anos

( ) 39 a 48 anos

( ) 49 a 59 anos

( ) mais de 59 anos

3. Escolaridade:

( ) Superior

( ) Pós graduado

( ) Mestrado

( ) Doutorado

Qual seu conhecimento acerca da hotelaria hospitalar e opiniões a respeito do tema?

Quais os componentes fazem parte da hotelaria hospitalar?

De que forma a hotelaria hospitalar influencia na recuperação do paciente?

Qual a influênciada hotelaria hospitalar em seu cotidiano de trabalho? 